RAD Conference Proceedings, vol. 2, pp. 186-190, 2017

www.rad-proceedings.org

novororitty

\title{
A PENCIL BEAM KERNEL MODEL FOR FLATTENING FILTER-FREE X-RAY BEAMS
}

\author{
Mehmet Ertuğrul Ertürk ${ }^{*}$, Cemil Kocar², Salih Gürdallı', Mehmet Tombakoğlu² \\ ${ }^{1}$ MNT Health Care, Beşiktaş, Istanbul, Turkey \\ ${ }^{2}$ Hacettepe University, Department of Nuclear Engineering, Beytepe, Ankara, Turkey
}

\begin{abstract}
Fast and accurate dose computation is an important requirement for algorithms that are often used in optimization schemes. Decreasing the number of variables and parameters and the amount of tabulated data can reduce computation time. Flattening Filter-Free (FFF) beams provide reduced profile shape variations with depth relative to flattened beams. Therefore, the pencil beam kernel of a FFF beam must exhibit the reduced variation with depth when compared to the kernel of flattened beams. In this paper, a kernel with a minimal number of parameters is derived for the FFF beams. Moreover, some of the parameters are defined as depth independent. A finite-size pencil beam dose calculation model was used for kernel generation. The grid size for the dose calculation was set to $2.5 \mathrm{~mm}$. During the kernel generation, the parameters (pre-exponential constants and exponential constants) of the kernel were determined in such a way that the difference between the computed and measured profiles is minimized by the global gamma analysis technique. The criteria for this technique were $1 \%$ dose difference at distance of $1 \mathrm{~mm}$ with a $10 \%$ threshold. Profiles for each field $\left(5 \times 5 \mathrm{~cm}^{2}, 10 \times 10 \mathrm{~cm}^{2}\right.$, and $\left.20 \times 20 \mathrm{~cm}^{2}\right)$ at five standard depths ( $d_{\max }, 5 \mathrm{~cm}, 10$ $\mathrm{cm}, 20 \mathrm{~cm}$, and $30 \mathrm{~cm}$ ), a total of 15 profiles, were used to generate the kernels. The multi-objective, non-derivative, unconstrained, non-linear optimization method in the programming package MATLAB (Mathworks, Natick, MA) optimization toolbox was used to generate kernel parameters. Commissioning of the model was performed for the static fields and the intensity-modulated radiation therapy (IMRT) fields. In static fields and dynamic IMRT fields, more than $95 \%$ of data points satisfied the criteria defined in the global gamma analysis with $3 \%$ and $3 \mathrm{~mm}$. There was a good agreement between modelled and measured data in both cases. It is demonstrated that the pencil beam model developed in this study could be used for FFF x-ray beams. Pencil beam kernel parameters do not need to be defined at each depth.
\end{abstract}

Key words: Dose computation, Flattening Filter Free, Kernel generation, Pencil Beam Kernel

DOI: $10.21175 /$ RadProc.2017.38

\section{INTRODUCTION}

Fast and accurate dose computation is an important requirement of radiotherapy. The dose calculation using pencil beam kernels was shown as an accurate algorithm to meet requirements for different cases such as calculation of dose distributions at irregular shaped fields [3], inhomogeneous medium [9], intensity-modulated radiation therapy (IMRT) fields [8, 9], or stereotactic radiosurgery (SRS) [7] modalities. Pencil beam model also defined as a fast algorithm $[3,4,8]$. Convolution of pencil beam kernel is a well- known dose calculation method, which has been studied since the mid 1980's [1, 2]. Various studies were performed to develop much accurate, fast and reliable dose calculation model $[3-9,12,14,15]$. Studies could be classified in different groups, such as the generation of kernel, improvement of the calculation model and adaptation of model to different energies or linear accelerators (linac).

Some studies generated kernels by utilizing Monte Carlo techniques [3, 4], while the others utilized analytic methods [4], and broad beam data [5, 6]. In this study, kernels are generated from the broad beam data. Kernels are also generated for different purposes, such as Dong et al. [7] developed a pencil beam model to calculate the dose for their in-house design stereotactic radio surgery (SRS) aperture. Jelén et al. $[8,9]$ developed a model that applied an optimization stage.

This study focuses on developing a pencil beam kernel that can be used to calculate arbitrarily shaped fields and IMRT fields for 6-MV flattening filter-free (FFF) beams when the field is shaped with a high definition multi leaf collimator (HDMLC) (Varian Medical Systems Inc., Palo Alto, CA.). FFF and HDMLC were studied as they are relatively new features in radiotherapy. Pencil beam model has wide usage area in radiotherapy and it is still being investigated [14]. Pencil beam can be used as fine dose calculation algorithm [15], coarse algorithm at an optimization stage $[8,9]$ or plan quality assurance algorithm [14]. There is only one study that combined FFF and pencil beam kernel was done by Azcona et.al. [14]. They generated pencil beam kernel with film dosimetry but not fitted to a function. This study suggests generation of kernel from broad beam data as

*mehmet.erturk@mnt.com.tr 
a sum of two exponentials. Kernels are generated with non-linear optimization [10] by utilizing gamma analysis method [11].

\section{METHODS}

Linacs generate $\mathrm{x}$-rays from decelerated electrons at target. The spatial distribution of the incident electrons could be assumed as Gaussian. Thus, the intensity profile of the generated photons has a Gaussian shape. Due to historical reasons, the cone shaped metal filters are mounted to linac to achieve the flat dose profile at a certain depth for a specified field size. This filtered linac energies are usually named as 6 MV, 10 MV or etc. Modification of beam intensity resulted in the modification of beam spectrum, which is a well-known phenomenon named radial beam hardening/softening. Furthermore, due to the spatial variation of energy spectrum, the dose varies spatially - this also resulted in the variation of dose profiles with depth. Change in profiles can be seen in Figure 1. Pencil beam kernel, which is a Green's function that transforms incoming photons to the absorbed dose, is assumed to be invariant at certain depth [3, 12]. To handle these adverse effects of the spatial variation of spectrum, kernels are defined for each depth.

Contrary, as shown in Figure 2, the variation of FFF dose profiles with depth are negligible relative to filtered beams. To understand this phenomenon, the variation of energy spectrum of $6 \mathrm{MV} \mathrm{l}$ with and without a flattening filter was generated with a Monte Carlo model. Spectrum of filtered $6 \mathrm{MV}$ is illustrated at Figure 3 and spectrum of FFF $6 \mathrm{MV}$ is illustrated at Figure 4. A $40 \mathrm{~cm} \times 40 \mathrm{~cm}$ open beam phasespace at a $100 \mathrm{~cm}$ distance from source was created for filtered 6 $\mathrm{MV}$ and $6 \mathrm{MV}$ FFF. Spectrums at centre (disc radius 5 $\mathrm{mm}$ ), at $9.5 \mathrm{~cm}$ (on a ring with thickness $5 \mathrm{~mm}$ ) away from centre and $20 \mathrm{~cm}$ away from centre (on a ring with thickness $5 \mathrm{~mm}$ ). To provide these figures, the phase space data provided by Varian were used with BEAMNRC [13].

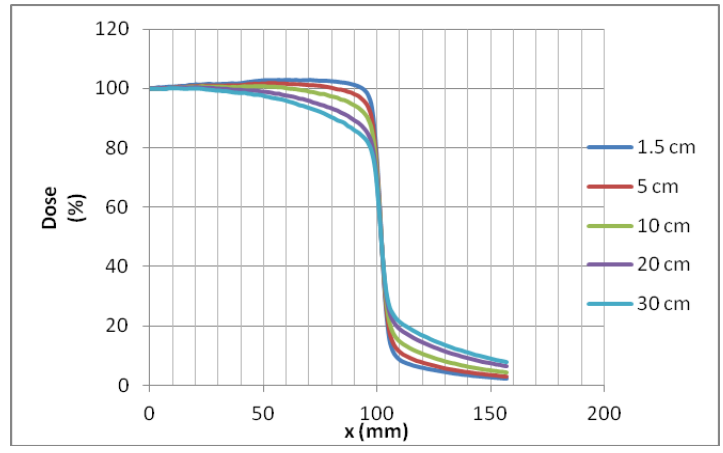

Figure 1. Dose profiles at five depths with flattening filter

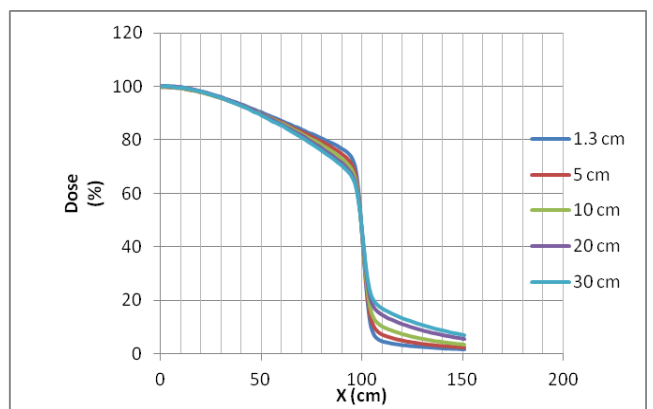

Figure 2. Flattening filter free dose profiles at five depths

These advances of FFF energies raised the question, which was investigated in this study, if it is possible to utilize the unvaried energy spectrum. As a rule of thumb, if the number of parameters in a pencil beam kernel model can be decreased, the computation time will be decreased. Thus, we tried to find a kernel with minimum number of parameters. The summation of the weighted exponentials in Equation 1, which is known as suitable pencil kernel, was used as a kernel function.

$$
K(r, z)=\sum_{i=1}^{N} A_{i} e^{-a_{i} r}
$$

Where $\mathrm{r}=\left(\mathrm{x}^{2}+\mathrm{y}^{2}\right)^{1 / 2}$, and " $\mathrm{K}(\mathrm{r}, \mathrm{z})$ " denotes the kernel value at distance of " $r$ " at depth " $z$ ". " $A_{i}$ " coefficient of the $\mathrm{i}^{\text {th }}$ exponential term and $\mathrm{a}_{\mathrm{i}}$ is the dose attenuation parameter. "N" is the number of exponents in Equation 1. Two exponents were used in the kernel model, where first term refers to the primary dose component and the second term is the scattered dose. Thus, Equation 1 becomes

$$
K(r, z)=A_{z} e^{-a_{z} r}+B_{z} e^{-b_{z} r}
$$

By defining $\mathrm{B}_{\mathrm{z}}$ as relative to primary ratio $\mathrm{A}_{\mathrm{z}}$ can be assumed as 1 and Equation 2 can be expressed as in Equation 3 .

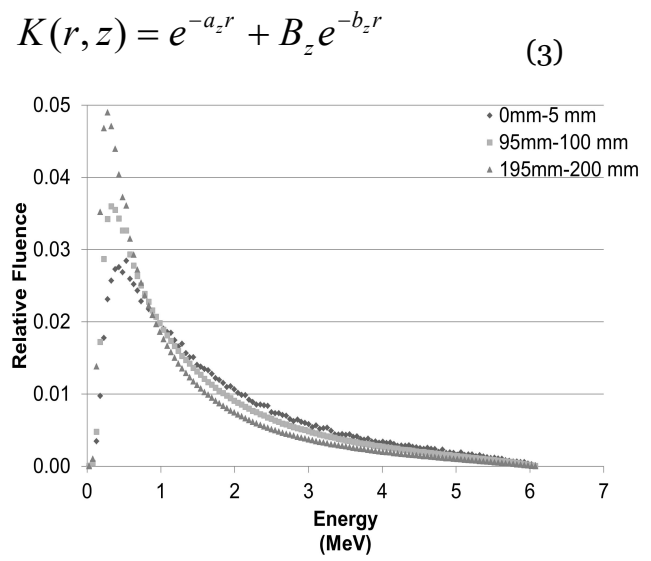

Figure 3. Variation in energy spectrum of $6 \mathrm{MV}$ with spatial position 


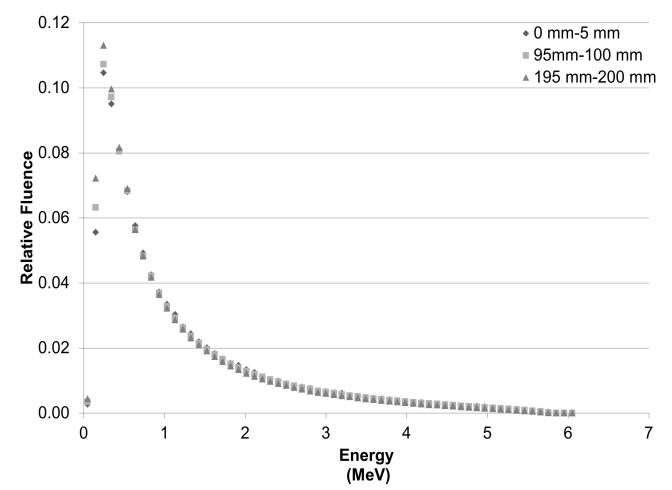

Figure 4. Variation in energy spectrum of $6 \mathrm{MV}$ FFF with spatial position

The off-axis ratio (OAR) of a point at a depth can be calculated with convolution integral of fluence and kernel of the depth, given in Equation 4.

$\operatorname{OAR}\left(x^{\prime}, y^{\prime}, z\right)=\int_{y_{1}}^{y_{2}} \int_{x_{1}}^{x_{2}} K\left(x \rightarrow x^{\prime}, y \rightarrow y^{\prime}, z\right) I(r, z) d x d y$ (4)

$\mathrm{I}(\mathrm{r})$ is the photon intensity at point r. OAR values, relative dose on profiles, less vary with depth at FFF relative to filtered energy. Therefore, the depth dependence of the attenuation parameters $\left(a_{z}, b_{z}\right)$ in Equation 3 could be ignored, and expressed as $(a, b)$. Finally, the kernel becomes (see Equation 5)

$$
K(r, z)=e^{-a r}+B_{z} e^{-b r}
$$

The kernel, presented in Equation 5, is used as the kernel, which has only one depth dependent parameter.

The diagonal profile measured at air was used as the intensity profile. This assumption made intensity map term "I(r, z)" independent from depth and transformed it to I $(r, 0)$. Thus, the OAR at any point at depth $\mathrm{z}$ is given in Equation 6.

$\operatorname{OAR}\left(x^{\prime}, y^{\prime}, z\right) \approx \int_{y_{1}}^{y_{2} x_{1}} \int_{x_{2}} K\left(x \rightarrow x^{\prime}, y \rightarrow y^{\prime}, z\right) I(x, y, 0) d x d y$

Calculations were performed on fan-line system and transformed to gantry system (see Equation 7,8 )

$$
\begin{aligned}
& x=x_{g} \frac{S S D+z}{S S D} \\
& y=y_{g} \frac{S S D+z}{S S D}
\end{aligned}
$$

$\mathrm{x}, \mathrm{y}$ : calculation grid size in fan line coordinate $\mathrm{xg}, \mathrm{yg}$ : calculation grid size in gantry coordinate SSD: Source surface distance and equal to $100 \mathrm{~cm}$ $\mathrm{z}$ : distance of rescaling layer to calculation layer

The kernel is generated from $6 \mathrm{MV}$ FFF broad beam data. Broad beam data are consisting dose profiles of three field, which are $5 \mathrm{~cm} \times 5 \mathrm{~cm}, 10 \mathrm{~cm} \times 10 \mathrm{~cm}, 20$ $\mathrm{cm} \times 20 \mathrm{~cm}$, at five depths, which are $1.3 \mathrm{~cm}, 5 \mathrm{~cm}, 10$ $\mathrm{cm}, 20 \mathrm{~cm}$ and $30 \mathrm{~cm}$. 15 profiles measured with a semiflex, 0.125 cc ion chamber (PTW, Freiburg, Germany). The kernel parameters were obtained via the multivariable, non-derivative, unconstrained, nonlinear optimization method developed by Lagarias et al. [10] in the programming package MATLAB (Mathworks, Natick, MA) optimization toolbox. Global Gamma analysis with the criteria of $1 \%$ and $1 \mathrm{~mm}$, as well as threshold of $10 \%$, was used as the penalty function of optimization.

A finite-size pencil beam dose calculation model was used for kernel generation. The grid size for the dose calculation was set to $2.5 \mathrm{~mm}$. During kernel generation, the parameters (pre-exponential constants and exponential constants) of the kernel were estimated such that the difference between the computed and measured profiles is minimized.

Measurement and calculation are the main two sources of uncertainty. To neglect fluctuation of linac dose rate a reference detector was used. To reduce uncertainty by increasing received signal measurement time was set to $0.4 \mathrm{sec}$. Detector used in the measurement has a 0.125 cc volume, which causes an inaccuracy at dose location especially in penumbra region. Other sources of uncertainty were numerical errors and optimization criteria during kernel generation. To decrease the numerical error $2.5 \mathrm{~mm}$ grid size was used during calculations and variable precision was to double precision.

\section{Calculations AND Results}

The comparison of measured profiles and modelled profiles were performed (see Figure 5). A total of 413 points on 15 profiles were analysed with global gamma analysis with the criteria of $1 \%$ and $1 \mathrm{~mm}$. Overall, 20 points failed in the region beyond a dose of $20 \%$, and 2 points failed between $100 \%$ and $50 \%$. The average value of gamma between $100 \%$ and $50 \%$ was 0.28 , and the average value of gamma for the entire system was 0.36 .
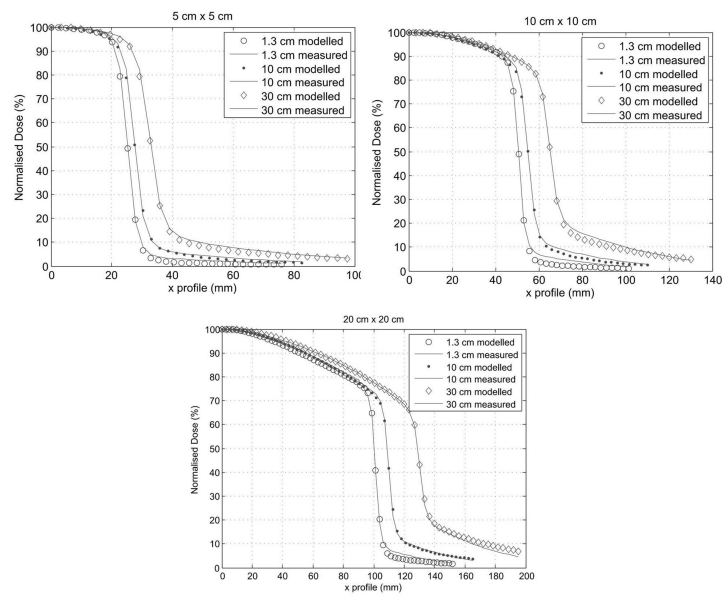

Figure 5. Comparison of various measured profiles with modelled profiles

The performance of the pencil beam model was tested with blocked fields and IMRT fields. To show the validity of algorithm in static fields and dynamic IMRT fields, static field and intensity maps for IMRT fields generated with Eclipse treatment planning system v.11.0.30 (Varian Medical Systems Inc., Palo Alto, CA). Thus, Eclipse treatment planning system was only used for generation of intensity map that required by our in- 
house algorithm as an input file. Generated C-shape and IMRT field's fluence maps were exported to a file which could be used as an input file of our algorithm. The shape of the blocked field is presented at Figure 6 . Truebeam STx (Varian Medical Systems Inc., Palo Alto, CA) with $6 \mathrm{MV}$ FFF was used for irradiation and measurement was done with diode array (MapCheck2, Sun Nuclear Corporation, Melburn, FL). Measured and calculated dose maps were compared with gamma analysis while the criteria were of $3 \%$ and $3 \mathrm{~mm}$, as well as a threshold of $10 \%$. The average gamma for cshaped field at 5 depths $(2 \mathrm{~cm}, 5 \mathrm{~cm}, 10 \mathrm{~cm}, 20 \mathrm{~cm}, 30$ $\mathrm{cm}$ ) is $97.8 \%$.
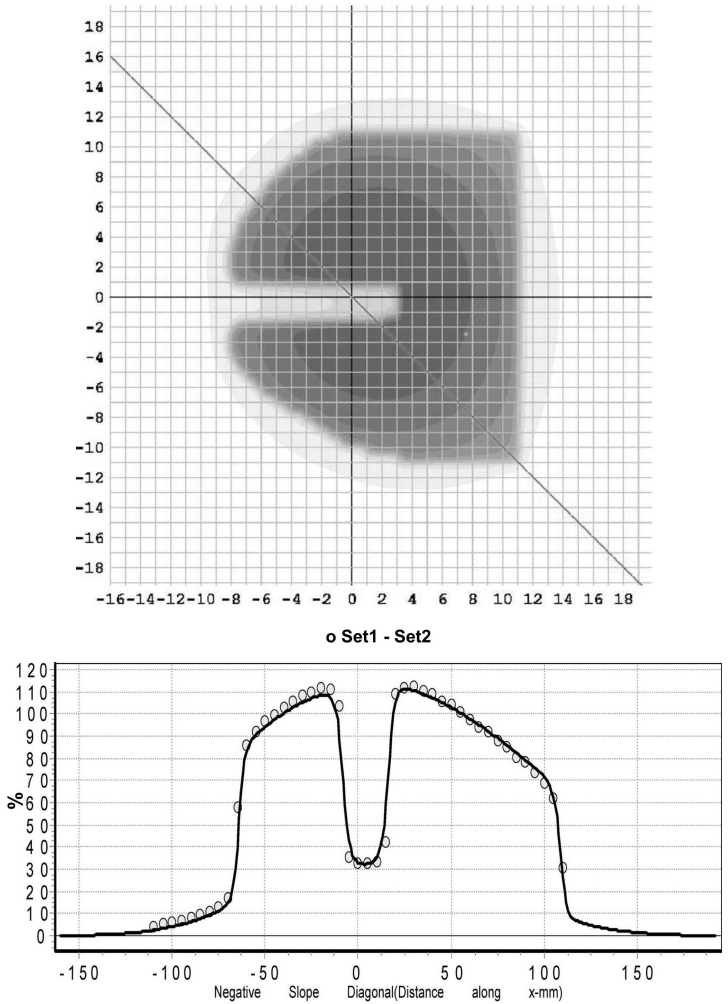

Figure 6. Comparison of measured and calculated dose at $20 \mathrm{~cm}$ depth
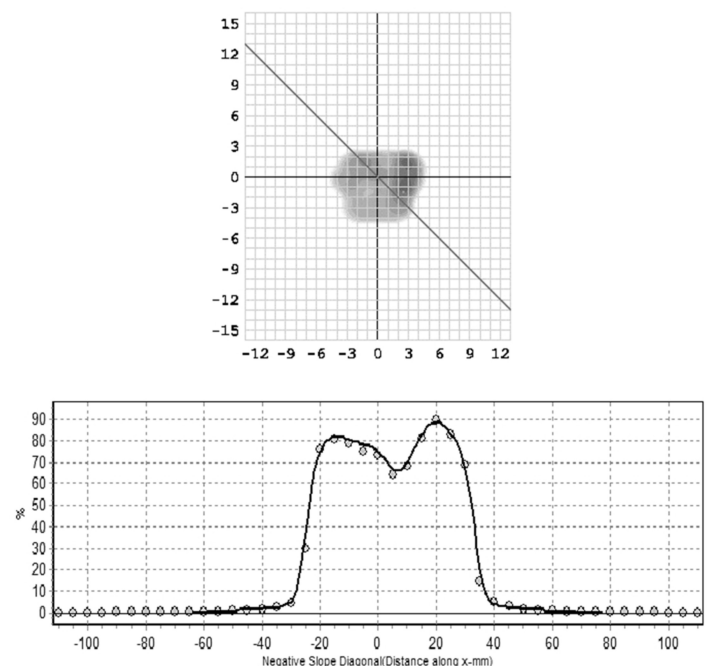

Figure 7. Comparison of calculated and measured IMRT field 189
Six dynamic IMRT fields were irradiated at $5 \mathrm{~cm}$ depth. All fields satisfied gamma passing rate of $95 \%$. The comparison of irradiated and calculated dynamic field is illustrated at Figure 7.

The coefficient $B_{z}$ was the only parameter that depended on depth and it was found that it changes linearly with depth (see Figure 8).

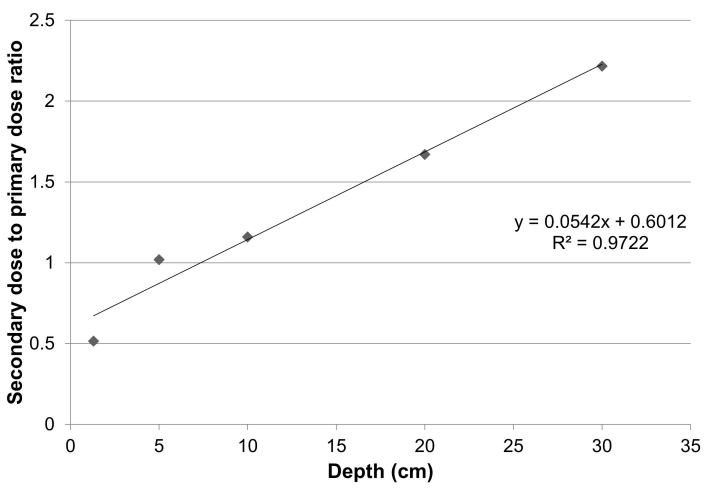

Figure 8. Variation of $\mathrm{B}_{\mathrm{z}}$ with depth

\section{CONCLUSION}

Calculated dose maps of static and dynamic cases have satisfied gamma analysis when compared with experimental data. Therefore, one can conclude that it is possible to simplify the pencil beam model for FFF beams by utilizing characteristics of FFF beams. Degreasing the number of parameters is shortening the computation time. Simplifying algorithm by utilizing beam characteristics can be implemented to other dose calculation algorithms. This approach is limited to FFF beams and implementing to a conventional energy may be resulted with improper dose calculation. Using the gamma analysis method as a penalty function during kernel generation is an effective approach. Kernel generation discussed by Azcona et.al. [14] was performed with film dosimetry. Data obtained during beam data measurements can be used purposed model in this paper. Methods used in this study can easily be applied to in-house researches, it requires fewer measurements. Kernels can also be generated as sum of two exponentials or as a function instead of tabulated data. Definition of kernels as function enables to create empiric expression. Simplification of algorithm by utilizing characteristics of FFF beam could be applied to other dose calculation algorithms. The performance of the algorithm for absolute dose distribution and definition of $\mathrm{B}_{\mathrm{z}}$ as a first order polynomial will be studied in future works. This approach can also be applied to inhomogeneous media in future work.

\section{REFERENCES}

1. A. Boyer and E. Mok, "A photon dose distribution model employing convolution calculations," Med. Phys., vol. 12, no. 2, pp. 169 - 177, Mar. 1985.

DOI: $10.1118 / 1.595772$

PMid: 4000072

2. A. L. Boyer, "Shortening the calculation time of photon dose distributions in an inhomogeneous medium," Med. Phys., vol. 11, no. 4, pp. 552 - 554, Jul. 1984. 
DOI: $10.1118 / 1.595526$

PMid: 6482848

3. R. Mohan and C.S. Chui, "Use of fast Fourier transforms in calculating dose distributions for irregularly shaped fields for three-dimensional treatment planning," Med. Phys., vol. 14, no. 1, pp. 70 77, Jan. 1987.

DOI: $10.1118 / 1.596097$ PMid: 3104741

4. A. Ahnesjö, M. Saxner and A. Trepp, "A pencil beam model for photon dose -calculation," Med. Phys., vol. 19, no. 2, pp. 263 - 273, Mar. 1992.

DOI: $10.1118 / 1.596856$ PMid: 1584117

5. C. S. Chui and R. Mohan, "Extraction of pencil beam kernels by the deconvolution method," Med. Phys., vol. 15 , no. 2, pp. 138 - 144, Mar. 1988.

DOI: $10.1118 / 1.596267$ PMid: 3386581

6. C.P. Ceberg, B.E. Bjärngard and T.C. Zhu, "Experimental determination of the dose kernel in high-energy x-ray beams," Med. Phy., vol. 23, no. 4, pp. 505 - 511, Apr. 1996. DOI: $10.1118 / 1.597807$

PMid: 9157261

7. L. Dong et al., "A pencil-beam photon dose algorithm for stereotactic radiosurgery using a miniature multileaf collimator," Med. Phys., vol. 25, no. 6, pp. $841-850$, Jun. 1998.

DOI: $10.1118 / 1.598294$

PMid: 9650171

8. U. Jeleń et al., "A finite size pencil beam for IMRT dose optimization," Phys. Med. Biol., vol. 50, no. 8, pp. 1747 - 1766, Apr. 2005.

DOI: $10.1088 / 0031-9155 / 50 / 8 / 009$

PMid: 15815094

9. U. Jeleń and M. Alber, "A finite size pencil beam algorithm for IMRT dose optimization: density corrections," Phys. Med. Biol., vol. 52, no. 3, pp. 617 633, Jan. 2007.

DOI: 10.1088/0031-9155/52/3/006

PMid: 17228109

10. J. C. Lagarias et al., "Convergence properties of the Nelder-Mead Simplex Method in low dimensions," SIAM Journal of Optimization, vol. 9, no. 1, pp. 112 - 147, Dec. 1998.

DOI: $10.1137 /$ S1052623496303470

11. D. A. Low and J. F. Dempsey, "Evaluation of the gamma dose distribution comparison method," Med. Phys., vol. 30, no. 9, pp. 2455 - 2464, Sep. 2003.

DOI: $10.1118 / 1.1598711$

PMid: 14528967

12. T. Bortfeld, W. Schlegel and B. Rhein, "Decomposition of pencil beam kernels for fast dose calculations in three-dimensional treatment planning," Med. Phys., vol. 20, no. 2, pp. $311-318$, Mar. 1993.

DOI: $10.1118 / 1.597070$

PMid: 8497215

13. D. W. Rogers, B. A. Faddegon, G. X. Ding, C. M. Ma, J. We, T. R. Mackie, "BEAM: A Monte Carlo code to simulate radiotherapy treatment units," Med. Phys., vol. 22, no. 5, pp. 503 - 524, May 1995

DOI: $10.1118 / 1.597552$

PMid: 7643786

14. J. D. Azcona et al., "Experimental pencil beam kernels derivation for $3 \mathrm{D}$ dose calculation in flattening filter free modulated fields," Phys. Med. Biol., vol. 61, no. 1, pp. $50-66$, Jan. 2016.

DOI: $10.1088 / 0031-9155 / 61 / 1 / 50$

PMid: 26611490

15. W. Ulmer, D. Harder, "A Triple Gaussian Pencil Beam Model for Photon Beam Treatment Planning," Z. Med Phys., vol. 5, no. 1, pp. 25 - 30, Jan. 1995. DOI: 10.1016/So939-3889(15)70758-o 